\title{
Perfil eletrocardiográfico dos atletas integrantes da equipe brasileira dos XI Jogos Paraolímpicos de Sydney 2000
}

\author{
Marcelo Bichels Leitão ${ }^{1}$
}

\section{RESUMO}

O esporte paraolímpico tem despertado o interesse da comunidade científica devido à diversidade de situações encontradas em seus atletas. Contudo, existe atualmente uma carência de informações sobre parâmetros clínicos e fisiológicos referentes a atletas portadores de deficiências. Este trabalho tem como objetivo descrever as características eletrocardiográficas encontradas nos atletas da delegação brasileira que participou dos XI Jogos Paraolímpicos de Sydney 2000. Foram avaliados 60 atletas, sendo 50 do sexo masculino e 10 do sexo feminino. Todos estes atletas foram submetidos a uma anamnese e a um exame físico, seguidos de eletrocardiograma de repouso (ECG), teste ergométrico (TE) e quando necessário de ecocardiograma (ECOC). De acordo com os resultados do ECG os indivíduos foram classificados em uma de três situações: ECG normal $(\mathrm{N}=31)$, ECG de Atleta $(\mathrm{N}=26)$ ou ECG anormal $(\mathrm{N}=3)$. Não foram observados eventos anormais no TE de nenhum atleta. Os três indivíduos que apresentaram ECG anormal foram submetidos a ECOC que se mostrou normal em todas as situações.

Palavras-chave: Eletrocardiograma. Atleta. Teste ergométrico. Paradesporto. Paraolimpíada. Deficientes.

\section{ABSTRACT}

\section{Electrocardiographic profile of the Brazilian team in the Sydney 2000 Paralympic Games}

Paralympic sports have attracted the interest of the scientific community as a consequence of the diversity of sit-

1. Médico Especialista em Medicina do Esporte. Mestrando em Cardiologia pela Universidade Federal do Paraná.

Submetido em: 4/4/02

Versão revisada recebida em: 1/5/02

Aceito em: 19/5/02

Endereço para correspondência:

Marcelo Bichels Leitão

Rua Gastão Câmara, 499, apto. 191

80730-300 - Curitiba, PR

E-mail leitaomb@uol.com.br uations found in such athletes. However, there still is a scarcity of information about clinical and physiological parameters of disabled athletes. This study describes the electrocardiographic characteristics observed in the athletes of Brazilian team that participated in the Sydney 2000 Paralympic Games. 60 subjects were evaluated, 10 female and 50 male. All of them were submitted to an anamnesis and a physical examination, followed by a rest electrocardiogram $(E C G)$, an exercise test (ET), and when needed, they were also submitted to an echocardiography (ECOC). According to the ECG results, subjects were classified as normal ECG $(N=31)$, athlete ECG $(N=26)$ or altered $E C G(N=3)$. No abnormalities were observed on ET. The three subjects who showed altered ECG were referred to ECOC, which was normal in all three cases.

Key words: Electrocardiography. Athlete. Exercise test. Disabled sports. Paralympic games. Disabled persons.

\section{INTRODUÇÃO}

A atividade física é sem sombra de dúvida um dos mais eficientes meios de promoção de saúde. O interesse pelo esporte competitivo tem aumentado expressivamente, e assim, jovens buscam através do sucesso esportivo sua realização pessoal e principalmente financeira. Estima-se que uma medalha de ouro olímpica proporcione a seu ganhador algo em torno de U\$ $1.000 .000,00$ em alguns países desenvolvidos.

Os médicos envolvidos no cuidado da saúde de indivíduos praticantes de atividades físicas e de equipes esportivas determinam o estado de saúde e a capacidade de treinar e competir destes através de uma avaliação pré-participação (APP).

Enquanto os atletas tendem a ver esta avaliação como uma mera formalidade que pode liberar ou impedir sua participação nos esportes, os profissionais de saúde devem valorizar a APP como uma intervenção importante que permite a participação segura em atividades esportivas.

Dentro da APP, a avaliação cardiológica tem grande importância, já que doenças cardiovasculares respondem por mais de $95 \%$ das causas de morte súbita em atletas jovens. 
Apesar de rara, a morte súbita em atletas, especialmente nos jovens, sempre causa uma grande impacto onde quer que ocorra, tanto pela grande repercussão na mídia quanto pela perplexidade que acomete a população devido à visão geral de que os atletas treinados constituem o segmento mais saudável da sociedade.

Além da detecção precoce de doença cardiovascular possibilitar a exclusão de atletas de alto risco para morte súbita cardíaca, em muitas circunstâncias ela permite também intervenções terapêuticas oportunas que podem prolongar a vida destes indivíduos.

Portanto, uma das principais finalidades da APP cardiológica é identificar indivíduos de alto risco cardiovascular que necessitem de investigação complementar. De acordo com os resultados desta investigação, os atletas podem ou ser liberados à prática de esportes competitivos sem restrição (com ou sem tratamento) ou ser recomendados a evitar determinadas modalidades esportivas ou ainda ser aconselhados a não se envolver com esportes competitivos.

$\mathrm{O}$ estabelecimento de um diagnóstico cardiovascular definitivo remete à aplicação das orientações da Conferência de Bethesda sobre participação de indivíduos portadores de cardiopatias em atividades físicas competitivas.

No âmbito nacional, a Sociedade Brasileira de Medicina do Esporte, em suas mais recentes publicações, tem invariavelmente destacado a importância da realização regular da APP.

$\mathrm{O}$ presente artigo tem como finalidade descrever a avaliação cardiológica e em especial o perfil eletrocardiográfico do grupo de atletas componentes da delegação brasileira que participou dos jogos paraolímpicos de Sydney 2000.

\section{MÉTODOS}

Foram avaliados os atletas convocados a integrar a equipe brasileira que iria disputar os Jogos Paraolímpicos de Sydney no ano de 2000. Esta avaliação foi realizada em duas fases distintas. A primeira ocorreu em Recife (PE) de 31 de julho a 4 de agosto de 2000 e a segunda em São Paulo (SP) entre 7 e 11 de agosto de 2000. O grupo que se submeteu à avaliação cardiológica completa se compunha de um total de 60 atletas, sendo que destes 50 eram do sexo masculino e 10 do sexo feminino. As características gerais do grupo, distribuição por modalidades esportivas e por diferentes deficiências se encontram à disposição em outros artigos desta separata.

\section{Metodologia de avaliação}

a) Anamnese: durante a realização da anamnese geral, foram desenvolvidas questões específicas dirigidas à iden- tificação de situações de risco de doença cardiovascular. Em todos os atletas avaliados, se encontrou na anamnese como dado positivo, apenas um atleta do tênis de mesa masculino com história pregressa de hipertensão arterial sistêmica.

b) Exame físico: consistiram do exame físico cardiológico a obtenção dos seguintes dados:

- pulso tomado em artéria radial, femoral ou carótida (nesta ordem de preferência de acordo com a deficiência que porventura o atleta apresentasse) com contagem em 60 segundos para se obter o número de impulsos por minuto;

- frequiência cardíaca obtida através de ausculta cardíaca com contagem em 60 segundos para se obter o número de sístoles ventriculares por minuto;

- pressão arterial obtida por método auscultatório com esfigmomanômetro aneróide, medida em milímetros de mercúrio ( $\mathrm{mm}$ de $\mathrm{Hg}$ ), utilizando as fases I e V de Korotkoff para identificar respectivamente a pressão arterial sistólica e diastólica;

Além disso, o atleta era submetido à inspeção, palpação e ausculta de região precordial, bem como à palpação de pulsos periféricos.

Não foram encontradas alterações significativas de dados obtidos através do exame físico cardiológico. $\mathrm{O}$ único atleta que se identificou na anamnese como portador de HAS estava sob uso regular de medicação anti-hipertensiva e apresentou níveis tensionais normais.

c) Eletrocardiograma de repouso: todos os atletas da equipe paraolímpica brasileira foram submetidos a um eletrocardiograma (ECG) de repouso.

O ECG foi realizado com os indivíduos deitados, obtendo-se as derivações precordiais habituais de V1 a V6. As derivações periféricas (DI, DII, DIII, aVR, aVL, aVF) também foram obtidas e quando se realizou exames de atletas com amputação de membro(s) se posicionou o eletrodo na raiz do(s) membro(s) amputado(s), ou na posição mais distal possível do coto de amputação, de acordo com a situação encontrada.

Para o ECG de repouso utilizou-se um software da marca HeartWare (Belo Horizonte, MG, Brasil) modelo Ergo98 versão 1.1.6.1.

Os traçados eram obtidos e avaliados na tela de um computador pessoal, e quando fossem identificados como de boa qualidade eram armazenados para posterior impressão e interpretação dos mesmos.

d) Eletrocardiograma de esforço: o eletrocardiograma de esforço foi realizado nos atletas avaliados nas duas etapas (Recife e São Paulo), realizando-se a monitorização em três derivações (CM5, aVF e V2). Não se utilizou nenhum protocolo específico habitualmente utilizado em er- 
gometria. A monitorização eletrocardiográfica de esforço foi realizada durante os testes de mensuração direta de $\dot{\mathrm{V}}_{2}$ máximo, sendo que a descrição dos protocolos utilizados encontra-se no capítulo específico sobre estas avaliações neste livro.

Nas avaliações realizadas em Recife (PE), utilizou-se sistema de monitorização eletrocardiográfica informatizado da marca Micromed. Já em São Paulo (SP) utilizou-se sistema de monitorização eletrocardiográfica informatizado da marca Marquette.

e) Outros métodos: devido a alterações encontradas em três atletas, conforme descrito a seguir na seção resultados, foram realizados nestes indivíduos ecocardiograma bidimensional com Doppler e mapeamento de fluxo em cores.

Não foi necessária a utilização de outros métodos complementares em cardiologia para elucidação de anormalidades encontradas neste grupo de indivíduos.

\section{RESULTADOS}

Os resultados das avaliações cardiológicas realizadas nos atletas integrantes da delegação brasileira para as Paraolimpíadas de Sydney 2000 são descritos a seguir.

O número total de atletas avaliados está exposto na figura 1.

Nestas avaliações, foi possível se observar alguns padrões de ECG que foram subdivididos da seguinte forma:

1) ECG normal, para aqueles que não apresentassem padrões diferentes do normal para a população geral não atleta;

2) ECG de atleta, para os que apresentassem padrões que diferissem dos encontrados na população geral não atleta, mas que fossem freqüentemente encontrados em indivíduos atletas e que pudessem ser explicados por ação de treinamento sistemático sobre o sistema cardiovascular;

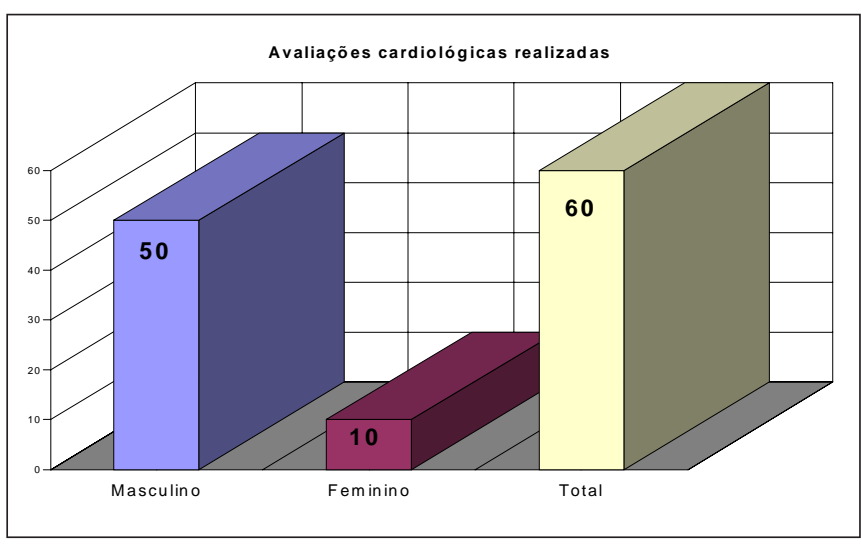

Fig. 1 - Número de avaliações cardiológicas realizadas
3) ECG anormal, quando as características encontradas, que diferissem do padrão encontrado na população geral não atleta, não pudessem ser explicadas pelo efeito do treinamento sistemático sobre o sistema cardiovascular;

A figura 2 mostra a distribuição dos ECG conforme a divisão comentada acima.

Dentre os traçados classificados como ECG de atleta, encontramos diversas características eletrocardiográficas que são habitualmente descritas na literatura como decorrentes de treinamento físico sistemático sobre o sistema cardiovascular. Estas características estão destacadas na figura 3 .

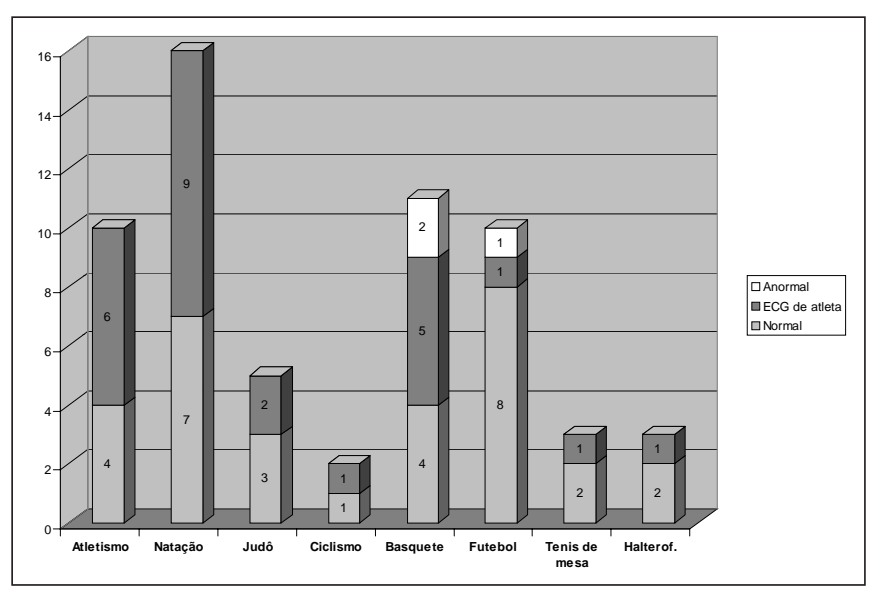

Fig. 2 - Distribuição dos resultados de ECG encontrados, subdivididos de acordo com a seguinte classificação: normal, ECG de atleta ou anormal. Resultados divididos por modalidade esportiva. Os números vistos em cada seção de cor diferente das barras expressam o valor absoluto de indivíduos de cada modalidade para cada classificação de ECG.

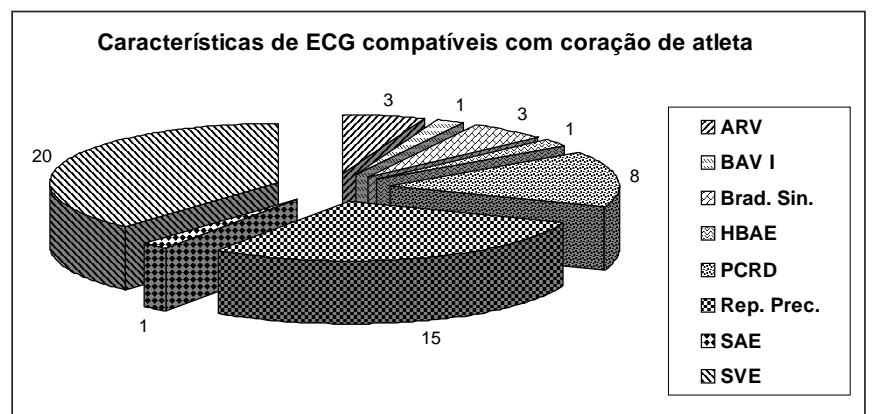

Fig. 3 - Valores absolutos das características de ECG classificadas como ECG de atleta. Em alguns traçados foi observada mais de uma alteração simultânea. Legenda: $\boldsymbol{A} \boldsymbol{R} \boldsymbol{V}$ - alteração primária da repolarização ventricular; BAV I - bloqueio atrioventricular de primeiro grau; $\boldsymbol{B r a d}$. Sin. - bradicardia sinusal; $\boldsymbol{H B A E}$ - bloqueio da divisão ântero-superior do ramo esquerdo do feixe de His; $\boldsymbol{P C R D}$ - perturbação da condução do ramo direito do feixe de His; Rep. Prec. - repolarização ventricular precoce; $\boldsymbol{S A E}$ - sobrecarga atrial esquerda; $\boldsymbol{S V E}$ - sobrecarga ventricular esquerda. 


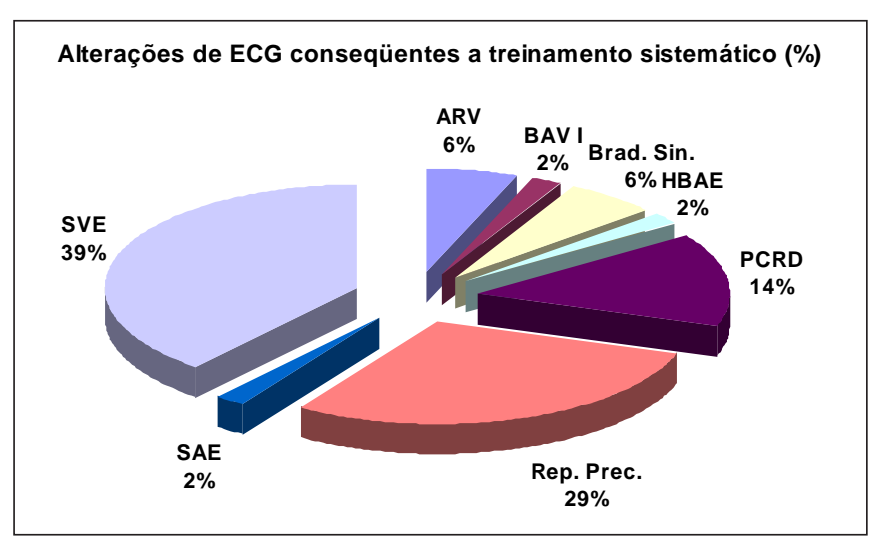

Fig. 4 - Percentuais das alterações de ECG classificadas como ECG de atleta em relação ao número total de atletas. Legenda: $\mathbf{A R V}$ - alteração primária da repolarização ventricular; BAVI - bloqueio atrioventricular de primeiro grau; Brad. Sin. - bradicardia sinusal; $\boldsymbol{H B A E}$ - bloqueio da divisão ântero-superior do ramo esquerdo do feixe de His; PCRD - perturbação da condução do ramo direito do feixe de His; $\boldsymbol{R e p}$. Prec. - repolarização ventricular precoce; $\boldsymbol{S A E}$ - sobrecarga atrial esquerda; $\boldsymbol{S} \boldsymbol{V E}$ - sobrecarga ventricular esquerda.

Na figura 4 podem-se observar estas características eletrocardiográficas e sua ocorrência percentual em relação ao total de atletas avaliados.

Observou-se também a distribuição destas características de ECG de acordo com o sexo dos atletas. Estes dados podem ser observados na figura 5 .

\section{DISCUSSÃO}

A divisão dos padrões de ECG encontrados como exposto acima se justifica devido a alta incidência de variações eletrocardiográficas encontradas em grupos de atletas, que apesar de não poderem ser consideradas normais quando observadas em indivíduos não atletas, tampouco remetem diretamente a um diagnóstico de doença cardiovascular se observadas em atletas.

Assim, seguindo recomendações de literatura, características eletrocardiográficas compatíveis com efeito de treinamento sistemático sobre o sistema cardiovascular, em indivíduos assintomáticos, com exame físico normal e sem história familiar pregressa significativa para cardiopatias e/ou morte súbita, não devem ser valorizadas e tampouco investigadas de forma mais aprofundada.

No grupo de atletas avaliados, a incidência deste tipo de características foi semelhante à de levantamentos publicados na literatura para distúrbios de condução intraventricular do estímulo elétrico (PCRD) e para sobrecarga ventricular esquerda (SVE). No entanto, a freqüência de ocorrência de alterações inespecíficas da repolarização ventricular (SVE), distúrbios da condução intra-atrial do estí-

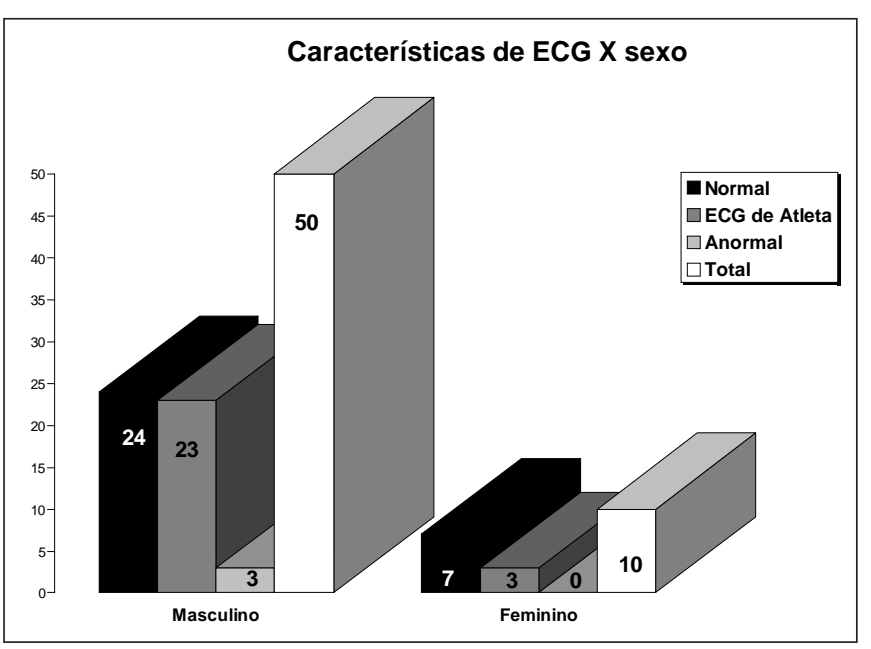

Fig. 5 - Distribuição dos resultados de ECG encontrados, subdivididos de acordo com a classificação: normal, ECG de atleta ou anormal. Resultados divididos por sexo. Os números em cada seção de cor diferente das barras expressam o valor absoluto de indivíduos por sexo para cada classificação de ECG.

mulo (BAV I), bradicardia sinusal, e repolarização ventricular precoce foram inferiores a levantamentos de ECG de equipes esportivas encontrados na literatura.

A comparação dos dados obtidos neste levantamento com dados de literatura se torna muitas vezes dificultada por uma série de fatores.

Primeiramente, a maioria das séries de levantamento de ECG de atletas existentes na literatura não são realizadas em equipes de portadores de deficiências físicas. É provável que a maioria das deficiências que ocorrem no grupo em estudo não interfiram nas características eletrocardiográficas encontradas, porém a carência de grandes estudos em populações de atletas portadores de deficiências impede a obtenção de conclusões mais definitivas.

Em segundo lugar, o número total de atletas avaliados já é por si só um número pequeno. Se subdividíssemos este grupo quanto a sexo, faixa etária, modalidade, tipo predominante de treinamento e tipo de deficiência, teríamos grupos compostos por número tão pequeno de atletas que por muitas vezes transformaríamos esta avaliação em mero relato de casos.

Finalmente, devemos ressaltar a heterogeneidade de situações que encontramos em relação ao grau de treinamento deste grupo. Se por um lado existem no grupo verdadeiros atletas, com programação de treinamentos adequada e sistematizada, no outro extremo encontramos indivíduos que muitas vezes tinham a oportunidade de treinar apenas uma vez por semana. Num país em que ser atleta já se constitui freqüentemente em motivo de aplauso, o paradesporto enfrenta as dificuldades dos atletas normais multiplicadas 
várias vezes. Portanto, a constatação de que neste grupo existem indivíduos que treinavam pouco ou quase nada simplesmente reflete a realidade do desporto adaptado no Brasil.

A realização de exames complementares cardiológicos não invasivos de rotina em populações esportivas é motivo de grande debate na literatura. Por um lado é indiscutível que a realização destes procedimentos aumenta a probabilidade de se detectar alguma doença cardiovascular em atletas. No outro extremo, levando-se em consideração a baixa prevalência de doenças cardiovasculares causadoras de morte súbita na população, a relação custo-benefício da inserção de determinados procedimentos na rotina das APP na maioria das vezes é desfavorável, desestimulando este tipo de procedimento.

No grupo em questão optou-se por desenvolver uma história clínica e exame físico detalhados, com ênfase em dados relevantes do sistema cardiovascular, associados a um ECG de repouso. A identificação de dados positivos que trouxessem a suspeição de anormalidades cardiovasculares estabeleceria a necessidade de investigações subseqüentes.

\section{REFERÊNCIAS}

1. Bjornstad H, Storstein 1, Meen HD, Hals O. Electrocardiographic findings in athletic students and sedentary controls. Cardiology 1991;79: 290-305.

2. Cantwell JD. Preparticipation physical evaluation: getting to the heart of the matter. Med Sci Sports Exerc 1998;30:s341-4.

3. Carvalho T, Nóbrega ACL, Lazzoli JK, Magni JRT, Rezende L, Drummond FA, et al. Posição oficial da Sociedade Brasileira de Medicina do Esporte: Atividade física e saúde. Rev Bras Med Esporte 1996;2:79-81.

4. Dyment PG. The preparticipation physical examination. In: Bar-Or O, editor. The child and adolescent athlete. $1^{\text {st }}$ ed. London: Blackwell Science 1996:243-59.

5. Fletcher GF. The athlete's electrocardiogram. In: Williams RA, editor. The athlete and heart disease diagnosis, evaluation $\&$ management. $1^{\text {st }}$ ed. Philadelphia: Lippincott Williams \& Wilkins, 1999:173-81.

6. Lazzoli JK, Nóbrega ACL, Carvalho T, Oliveira MAB, Teixeira JAC Leitão MB, et al. Posicionamento oficial da Sociedade Brasileira de Medicina do Esporte: Atividade física e saúde na infância e adolescência. Rev Bras Med Esporte 1998;4:107-9.

7. Lazzoli JK, Oliveira MAB, Leitão MB, Nóbrega ACL, Nahas RM, Rezende L, et al. Posicionamento oficial da Sociedade Brasileira de Medi-

\section{CONCLUSÕES}

Desta forma, do ponto de vista cardiovascular, entendemos que os atletas integrantes da equipe brasileira que participou dos jogos paraolímpicos de Sydney 2000 tiveram uma avaliação médica cardiológica adequada, não demonstrando nenhuma situação grave que impedisse a participação de qualquer um deles nas competições. Além disso, os achados encontrados nesta avaliação foram semelhantes aos descritos em levantamentos encontrados na literatura.

Propõe-se que este tipo de trabalho seja continuado e ampliado para que futuramente se tenha a possibilidade de, com um maior número de atletas avaliados, se estabelecer um perfil cardiológico mais completo dos praticantes de desportos adaptados no Brasil.

\section{AGRADECIMENTOS}

O autor gostaria de agradecer a Doutora Andressa Miguel Leitão pela colaboração na revisão do texto e nas sugestões que enriqueceram o formato final deste trabalho, Comitê Paraolímpico Brasileiro (СРB), Secretaria Nacional de Esportes, Rede Cenesp/ Unifesp, Associação Fundo de Incentivo à Psicofarmacologia (Afip).

cina do Esporte: Esporte competitivo em indivíduos acima de 35 anos. Rev Bras Med Esporte 2001;7:83-92.

8. MacAuley D. Does preseason screening for cardiac disease really work? The British perspective. Med Sci Sports Exerc 1998;30:s345-50.

9. Maron BJ. Cardiovascular preparticipation screening of competitive athletes. In: Williams RA, editor. The athlete and heart disease diagnosis, evaluation \& management. $1^{\text {st }}$ ed. Philadelphia: Lippincott Williams \& Wilkins, 1999:273-84.

10. Mitchell JH, Maron BJ, Raven PB. American College of Sports Medicine/American College of Cardiology $26^{\text {th }}$ Bethesda Conference: Recommendations for determining eligibility for competition in athletes with cardiovascular abnormalities. J Am Coll Cardiol 1994;24:845-99.

11. Nóbrega ACL, Freitas EV, Oliveira MAB, Leitão MB, Lazzoli JK, Nahas RM, et al. Posicionamento oficial da Sociedade Brasileira de Medicina do Esporte e da Sociedade Brasileira de Geriatria e Gerontologia: Atividade física e saúde no idoso. Rev Bras Med Esporte 1999;5:207-11.

12. Pate RR, Pratt M, Blair SN, Haskell WL, Macera CA, Bouchard C. Physical activity and public health. A recommendation from the Centers for Disease Control and Prevention and the American College of Sports Medicine. JAMA 1995;273:402-7. 